\title{
The Regression Analysis of Sampling Linearity on Digital Single Board of TD Network by MINITAB
}

\author{
Wei He ${ }^{* 1}$ and Hui Wang ${ }^{2}$ \\ ${ }^{1}$ Shaanxi University of Technology, School of Physics and Electronic Information \\ Engineering, Shaanxi, Hanzhong, China, 723001 \\ ${ }^{2}$ Shaanxi University of Technology, School of Foreign Studies, Shaanxi, \\ Hanzhong, China, 723001 \\ E-mail:1263236559@qq.com
}

\begin{abstract}
As a two-way amplifier, TMB is located on the top of the tower outdoors, it plays an indispensable part in TD station. Besides amplifying the signals in the Radio-Frequency channels, TMB transmits pilot signals, detection signals and power signals between base stations. This article verifies involved indicators on one single board with regression analysis method based on previous tests of digital board and RF board in TMB. To improve prediction and accuracy in controlling, this article illustrates the interdependent relationship between input voltage of digital single board and sampling value by a deep analysis of simple linear regression and the mathematical model.
\end{abstract}

Keywords: Digital single board; regression model; voltage; sampling value

\section{Introduction}

TD [1-2] is the first international standard with independent intellectual property, creating a precedent for China's participation in international telecommunication standardization. TD is one of the international mainstream $3 \mathrm{G}$ standards. TD proposed standard, China's telecommunications industry is a model of technological innovation, but also China's third generation mobile communication development made important contributions. TD outdoor overhead bidirectional amplifier TMB TD is an important part of the chamber, providing a channel for the reception and transmission of the base transceiver station and a mobile station. For receiving it, TMB low noise amplification. It can guarantee the overall receiver noise figure channel; simultaneously, TMB [3] to the base station transmitter to amplify the signal, he can ensure that the distance covered TD system. TMB, in addition to completing the RF channel other than the signal amplification, and also transmits control signal, the detection signal and the power signal with the base stations. Therefore, monitoring and verification of the running boards and TMB digital RF board data is essential to facilitate the system to keep abreast of the working conditions of TMB.

Regression analysis [4-6] is an analysis of the number of dependencies objective things. It is a mathematical statistics commonly used method, which is to find the mathematical relationship is not entirely sure of the variables and statistical inference, a mathematical method of handling relationships between multiple variables. According to the experimental collection of digital input board power module voltage data, related indicators for digital single board, verified by Minitab software [1,7-10] Regression

* Corresponding Author 
analysis, the paper comes to a conclusion that the digital veneer of input voltage and sampling values of showed a single linear relation.

\section{The Regression Equation}

\subsection{Linear Regression Equation}

Among the correlations of variables, the simplest is linear relationship. Assuming a linear relationship exists between random variables $\eta$ and $\xi$, variable data obtained from the test point $\left(x_{i}, y_{i}\right)(i=1,2, \cdots, n)$ will be scattered around a straight line. Therefore, we can conclude that $\eta$ about the return type of the function $\xi$ is a linear function, that is $\mu(x)=a+b x$. With the following estimated parameters $a, b$ by the least squares method, assuming $y_{i}$ following a normal distribution $N\left(a+b x_{i}, \sigma^{2}\right)(i=1,2, \cdots, n)$, seek the partial derivative of $S=\sum_{i=1}^{n}\left(y_{i}-a-b x_{i}\right)^{2}$ to $a, b$ respectively, and make them equal to zero, then get the equations

$$
\left\{\begin{array}{c}
n a+\left(\sum_{i=1}^{n} x_{i}\right) b=\sum_{i=1}^{n} y_{i} \\
\left(\sum_{i=1}^{n} x_{i}\right) a+\left(\sum_{i=1}^{n} x_{i}^{2}\right) b=\sum_{i=1}^{n} x_{i} y_{i}
\end{array}\right.
$$

Solution

$$
\left\{\begin{array}{l}
\hat{a}=\bar{y}-\hat{b} \bar{x} \\
\hat{b}=l_{x y} / l_{x x}
\end{array}\right.
$$

Among which, $\bar{x}=\frac{1}{n} \sum_{i=1}^{n} x_{i}, \bar{y}=\frac{1}{n} \sum_{i=1}^{n} y_{i}, l_{x y}=\sum_{i=1}^{n} x_{i} y_{i}-n \bar{x} \bar{y}, l_{x x}=n s_{x}{ }^{2}$, and $s_{x}{ }^{2}$ is the Sample variance of observations $x_{1}, x_{2}, \cdots x_{n}$.

The linear equation $\hat{y}=\hat{a}+\hat{b} x$ is called a linear regression equation of $\eta$ on $\xi$, and $\hat{b}$ is regression coefficient, and the corresponding line is a regression line [11-13] .

\subsection{Linear Regression Analysis of Variance}

Assumes the linear regression equation of $\eta$ on $\xi$ is $\hat{y}=a+b x$, it is obvious that only if the regression coefficient $b \neq 0$, there exists a linear relationship between $\eta$ and $\xi$. Therefore, in order to test its prominence, we should test $H_{0}: b=0 ; H_{1}: b \neq 0$.

Consider the sum of squares $S_{T}=\sum_{i=1}^{n}\left(y_{i}-\bar{y}\right)^{2}$ about the observations $y_{1}, y_{2}, \cdots, y_{n}$. It indicates the general degree of dispersion about them, and 
Because $\hat{y}_{i}=\hat{a}+\hat{b} x_{i}, \hat{a}=\bar{y}-\hat{b} \bar{x}, \hat{b}=l_{x y} / l_{x x}$,

$$
\begin{gathered}
\begin{aligned}
& \sum_{i=1}^{n}\left(\hat{y}_{i}-\bar{y}\right)\left(y_{i}-\hat{y}_{i}\right)=\sum_{i=1}^{n} \hat{b}\left(x_{i}-\bar{x}\right)\left[y_{i}-\bar{y}-\hat{b}\left(x_{i}-\bar{x}\right)\right] \\
&=\hat{b}\left[\sum_{i=1}^{n}\left(x_{i}-\bar{x}\right)\left(y_{i}-\bar{y}\right)-\hat{b} \sum_{i=1}^{n}\left(x_{i}-\bar{x}\right)^{2}\right] \\
&=\hat{b}\left(l_{x y}-\frac{l_{x y}}{l_{x x}} l_{x x}\right) \\
& S_{T}=\sum_{i=1}^{n}\left[\left(\hat{y}_{i}-\bar{y}\right)+\left(y_{i}-\hat{y}_{i}\right)\right]^{2}
\end{aligned}
\end{gathered}
$$

2.3.

$$
=\sum_{i=1}^{n}\left(\hat{y}_{i}-\bar{y}\right)^{2}+\sum_{i=1}^{n}\left(y_{i}-\hat{y}_{i}\right)^{2}+2 \sum_{i=1}^{n}\left(\hat{y}_{i}-\bar{y}\right)\left(y_{i}-\hat{y}_{i}\right) \quad \begin{aligned}
& \text { The } \\
& \text { Prominence } \\
& \text { Test about }
\end{aligned}
$$

\section{Linear Correlation}

Therefore, $S_{T}=\sum_{i=1}^{n}\left(\hat{y}_{i}-\bar{y}\right)^{2}+\sum_{i=1}^{n}\left(y_{i}-\hat{y}_{i}\right)^{2}=S_{R}+S_{e}$.

Among which, $S_{R}=\sum_{i=1}^{n}\left(\hat{y}_{i}-\bar{y}\right)^{2}$ is called a regression sum of squares. Because $\frac{1}{n} \sum_{i=1}^{n} \hat{y}_{i}=\sum_{i=1}^{n}(\hat{a}+\hat{b} x)=\hat{a}+\hat{b} \bar{x}=\bar{y}, S_{R}$ is a deviation sum of squares of regression value $\hat{y}_{1}, \hat{y}_{2}, \cdots, \hat{y}_{n}$, which indicates the dispersion degree of it. And this dispersion is due to the corresponding changes in their regression line $x_{1}, x_{2}, \cdots x_{n}$, which is better performed with the following equation

$$
S_{R}=\sum_{i=1}^{n}\left(\hat{y}_{i}-\bar{y}\right)^{2}=\sum_{i=1}^{n}\left[\hat{b}\left(x_{i}-\bar{x}\right)\right]^{2}
$$

Therefore, $S_{R}$ indicates the linear correlation[14-17] between $\eta$ and $\xi$.

And $S_{e}=\sum_{i=1}^{n}\left(y_{i}-\hat{y}_{i}\right)^{2}$ is called the residual sum of squares, which is the min of $S=\sum_{i=1}^{n}\left(y_{i}-a-b x_{i}\right)^{2}$, and reflects the degree of deviation from the regression line of the observations $y_{1}, y_{2}, \cdots, y_{n}$. And its deviation results from the random factors beyond the linear impacts of $\xi$ towards $\eta$.

If the null hypothesis $H_{0}$ is right, we can get $\frac{S_{T}}{\sigma^{2}} \sim x^{2}(n-1) ; \frac{S_{R}}{\sigma^{2}} \sim x^{2}(1)$; $\frac{S_{e}}{\sigma^{2}} \sim x^{2}(n-2)$.And $S_{R}$ is independent to $S_{e}$. Therefore, the statistics $F=S_{R} /\left(S_{e} /(n-2)\right)$ is obedient to $\mathrm{F}$ distribution with the degree of freedom $(1, \mathrm{n}-2)$. 


\subsection{Introduction of Minitab}

Minitab [18-] is statistical analysis software. It can be used for learning about statistics as well as statistical research. Statistical analysis computer applications have the advantage of being accurate, reliable, and generally faster than computing statistics and drawing graphs by hand. Minitab is relatively easy to use once you know a few fundamentals.

For this example, we will draw a histogram and box plot of the temperature data and a scatter plot of the water consumption versus the temperature.

(1) To draw a histogram, select GRAPH > HISTOGRAM.

(2) Choose Simple and click OK.

(3) In the Graph Variables box, select $\mathrm{C} 1$ (Temperature).

(4) Click OK.

(6) Compare your answer with the resulting histogram shown on the right. (Note: You can change the settings for the width of the bars in the histogram by clicking the $\mathrm{x}$ axis and clicking EDITOR > EDIT X-Scale and then selecting the Binning tab).

(7) To draw a box plot, select GRAPH > BOXPLOT.

(8) Choose Simple under One Y and click OK.

(Note: If your data is broken down into categories, choose another type of box plot. For example if you were graphing GPA by Gender, you would choose With Groups to get two box plots, one for each gender.)

(9) In the Graph Variables, select C1 (Temperature).

(10) Click OK.

(11) Compare your answer with the resulting box plot shown on the right.

(12) To graph a scatter plot for water consumption based on temperature, select GRAPH > SCATTERPLOT.

(13) Choose Simple, and Click OK.

(14) In the first row, under Y, select C2 (Water Consumption) and under X, select C1 (Temperature).

(15) Click OK.

(16) Compare your graph with the graph shown on the right. 


\section{Experimental Data Acquisition and Analysis}

\subsection{The Sampling Port Test Block Diagram of Digital Single Board}

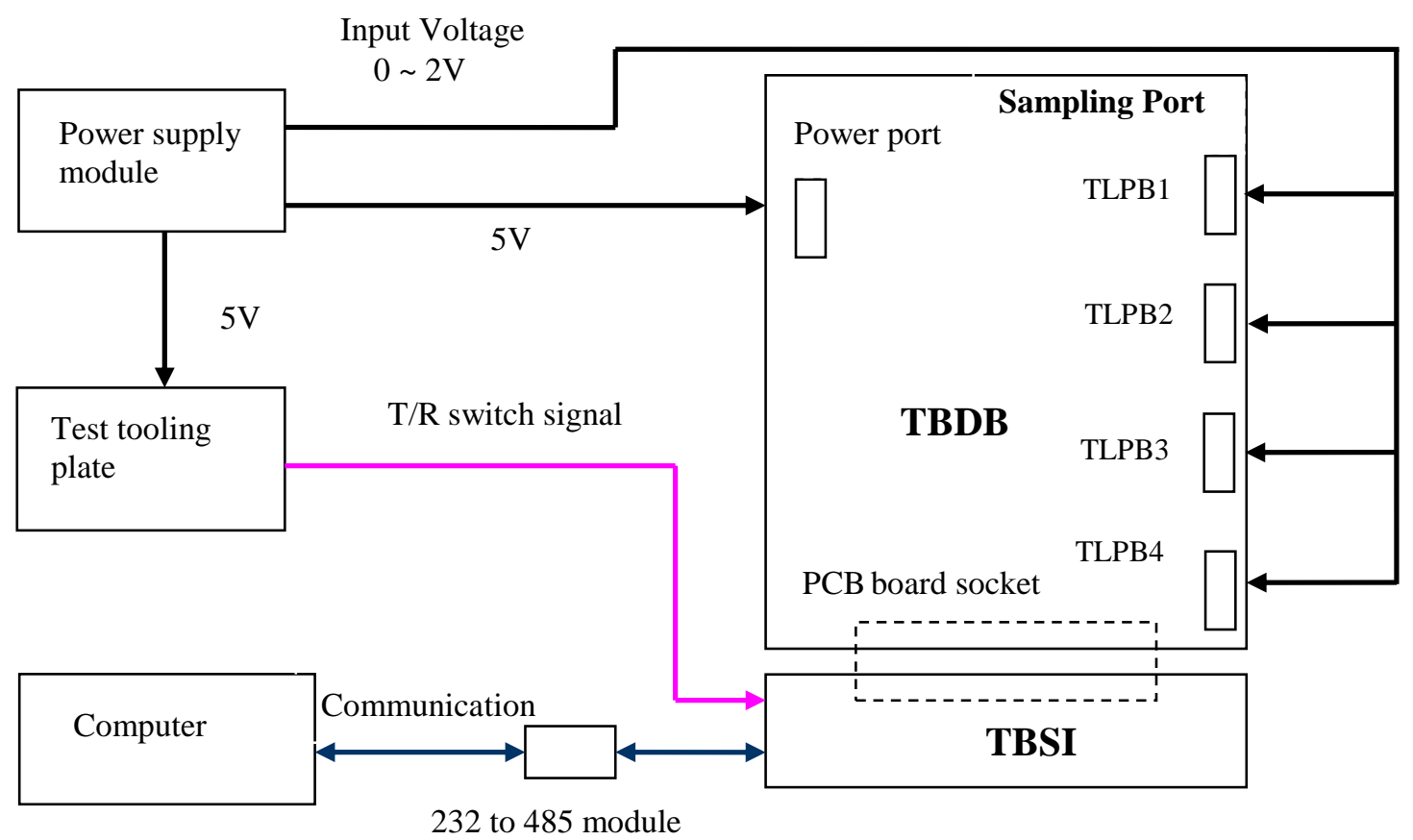

Diagram 1. The Test Block Diagram of the Object

Brief description: TBDB samples the input voltage $(0 \sim 2 \mathrm{~V})$ of the power supply module by TLPB1 TLPB4 Ports.

\subsection{Data Acquisition}

The data is collected with FLUKE 45. The ground of the input source is caught to Foot 1 of TLPB1 Port. The test point of the signal terminal is in Foot 5 of the single board socket. The sampling detection value of $\mathrm{AD}$ is got by the background software reading the detection value of "VSWR detection value" in the first channel.

Table 1. Test Record

\begin{tabular}{|c|c|c|c|c|c|c|c|c|}
\hline $\begin{array}{c}\text { Input Source } \\
\text { Value } \\
\text { Uin }(\mathrm{mV}) \\
\end{array}$ & 0.00 & 260.1 & 510.1 & 805.4 & 1023 & 1217 & 1490 & 1660 \\
\hline $\begin{array}{c}\text { AD } \\
\text { Sampling } \\
\text { Detection } \\
\text { Value AD } \\
\text { (V) }\end{array}$ & 0.000 & 0.242 & 0.500 & 0.797 & 1.008 & 1.203 & 1.477 & 1.648 \\
\hline $\begin{array}{l}\text { Input Source } \\
\text { Value } \\
\text { Uin }(\mathrm{mV})\end{array}$ & 1842 & 1927 & 2025 & & & & & \\
\hline $\begin{array}{c}\text { AD } \\
\text { Sampling } \\
\text { Detection } \\
\text { Value AD } \\
\text { (V) }\end{array}$ & 1.828 & 1.914 & 1.992 & & & & & \\
\hline
\end{tabular}




\subsection{Data Analysis}

(1) Calculation of the correlation coefficient

Correlations: AD, Uin

Pearson correlation of AD and Uin $=1.000$

$\mathrm{P}$-Value $=0.000$

If the correlation coefficient $=1$, it will indicate they are fully correlated.

(2) After analyzing regression with Minitab (Regression), recording residuals (Residuals), the results obtained are shown in Diagram 2 and Tables 2 4:

\section{Residual Plots for AD}
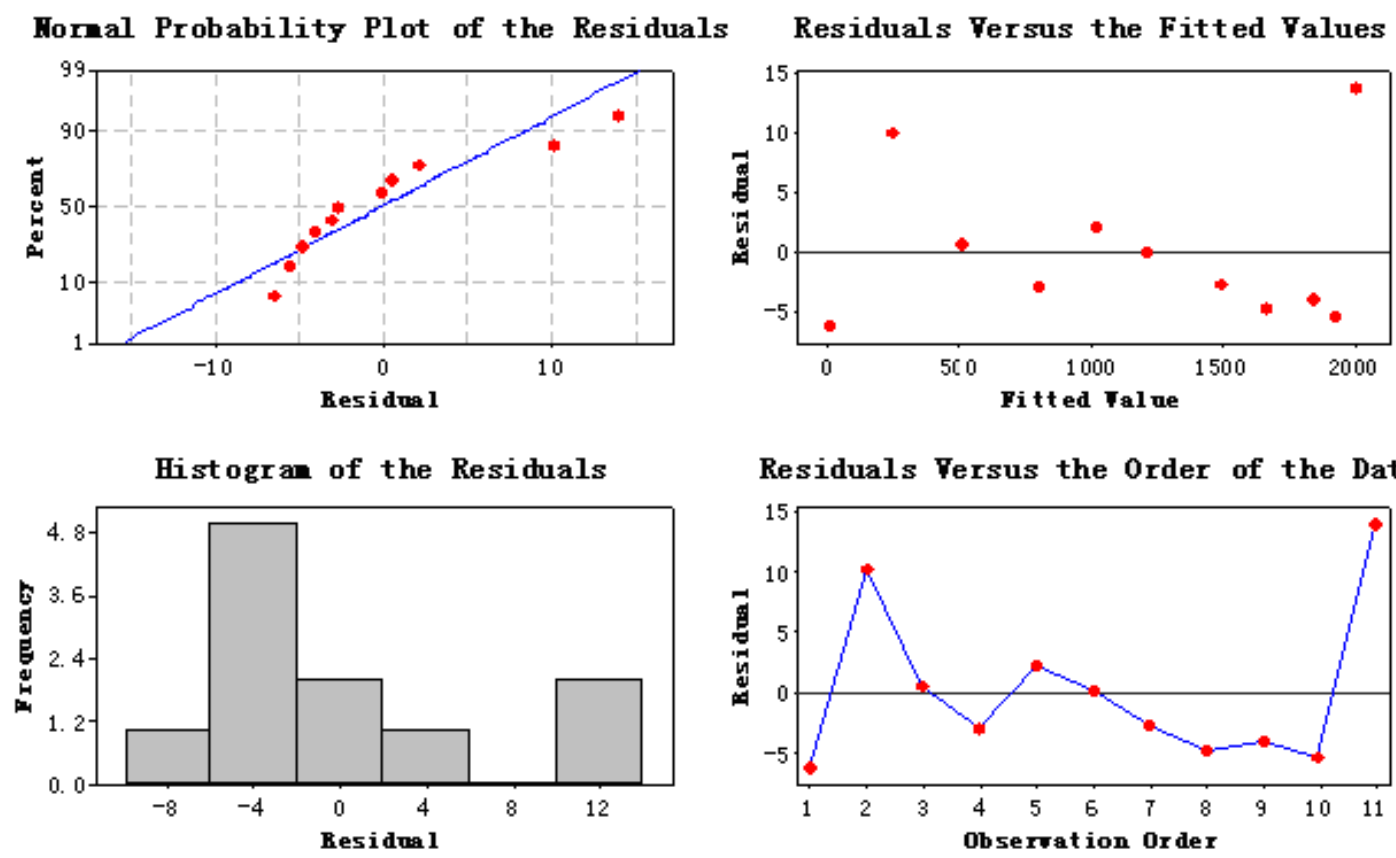

Residuals Versus the Order of the Data

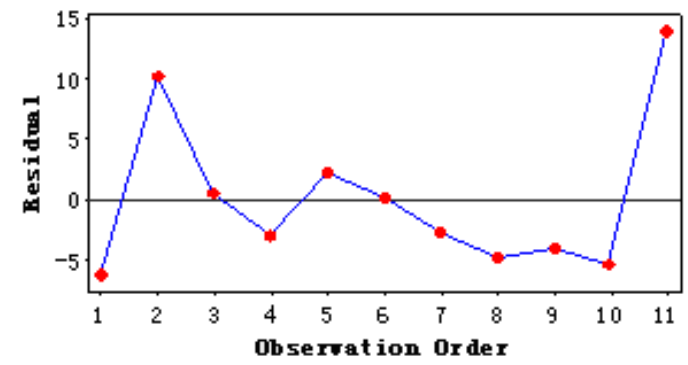

\section{Diagram 2. Analysis Diagram}

In Diagram 2, the top left displays the residuals to normal. The top right displays the residuals to fitted values. Residuals are randomly present in the vicinity of zero. The bottom right displays the residuals not exceeding the management limit.

The regression equation between $\mathrm{AD}$ and Uin should be

$$
\mathrm{AD}=6.41+1006 \mathrm{Uin}
$$

In it, 1006 is the slope coefficient.

Table 2. Regression Analysis

\begin{tabular}{ccccc}
\hline Arguments & Coefficient & $\begin{array}{c}\text { Coefficient } \\
\text { Standard Error }\end{array}$ & $\mathrm{T}$ & Probability \\
\hline Constant & 6.409 & 4.174 & 1.54 & 0.159 \\
Uin & 1006.35 & 3.15 & 319.16 & 0.000 \\
\hline
\end{tabular}

$\mathrm{S}=6.92544, \mathrm{R}-\mathrm{Sq}$ (forecast) $=100.0 \%, \mathrm{R}-\mathrm{Sq}$ (adjustment) $=100.0 \%$. The relation to Uin explains $100 \%$ of the variation about the $y$ values 
Table 3. Variance Analysis

\begin{tabular}{cccccc}
\hline Source & DOF & Sum of Squares & Mean Square & F Value & Probability \\
\hline Regression & 1 & 4885656 & 4885656 & 101865.60 & 0.000 \\
Residual & 9 & 432 & 48 & & \\
Error & 10 & 4886087 & & & \\
Sum & & & & \\
\hline
\end{tabular}

In Minitab, $\mathrm{P}$ values are an overall prominence test of the regression equation. If $\mathrm{P}<0.05$, reject $\mathrm{H} 0$, it indicates the regression relation is obvious in statistics.

Table 4. Outlier Analysis

\begin{tabular}{ccccccc}
\hline $\begin{array}{c}\text { Observation } \\
\text { Point }\end{array}$ & Uin & AD & $\begin{array}{c}\text { Fitted } \\
\text { Values }\end{array}$ & $\begin{array}{c}S_{e} \text { Fitted } \\
\text { Values }\end{array}$ & Residuals & $S_{T}$ Residuals \\
\hline 11 & 1.99 & 2025.00 & 2011.06 & 3.39 & 13.94 & $2.31 \mathrm{R}$ \\
\hline
\end{tabular}

The abnormal observation point, with the sense of physics, resulted from the A/D sampling saturation.

According to the results above, if $\mathrm{P}=0<0.05$, it's obvious, indicating the rejection of the null hypothesis, i.e., there is a linear relationship between $\mathrm{AD}$ and Uin. If $\mathrm{R}-\mathrm{Sq}$ $($ forecast $)=100.0 \%, \mathrm{R}-\mathrm{Sq}$ (adjustment) $=100.0 \%$, there will be a good fitted degree. In Plot 11, an abnormal data results from the saturation when sampling of $\mathrm{AD}$ gets close to $2 \mathrm{~V}$, which is entirely consistent with the circuit design.

\subsection{Conclusion}

The input voltage and the sampling values of TBDB digital single board show a unitary linear relationship.

Its corresponding regression equation should be $\mathrm{AD}=6.41+1006 \mathrm{Uin}$.

Note: the constant term of $6.41(\mathrm{mV})$ in the equation results from the level of reference sites when sampling A/D in the zero point.

\subsection{The Scatterplot Analysis}

Based on the tables above, the sampling scatter plot graph should be:

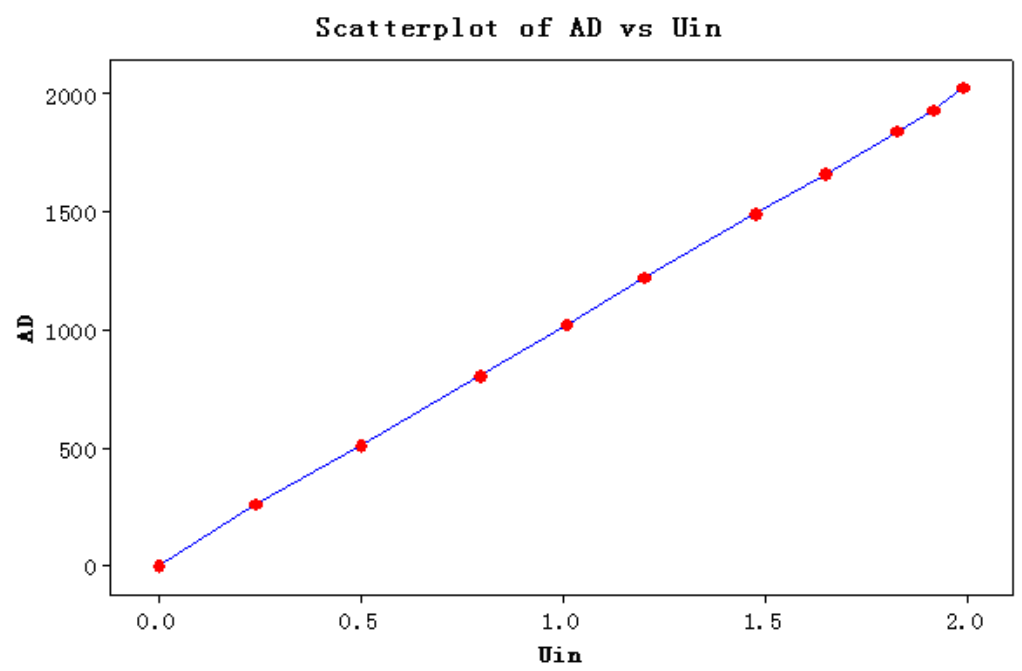

Diagram 3. The Sampling Scatter Plot Graph 
According to Diagram 3, in the input source ranging from 0 to $2 \mathrm{~V}$, the single board sampling shows high linearity (based on the previous calculation, its confidence gets 100 $\%$, which is entirely consistent with the real situation).

\section{Acknowledgements}

Project: the Science and Technology Program of the Education Department of Shaanxi Province Government (number: 2013JK1112). the Key Scientific Research Foundation of Shaanxi University of Technology(number: SLGKY14-29). the Key Scientific Research Foundation of Shaanxi University of Technology(number: SLGKY14-06).

\section{References}

[1] C. J. Shuai, "The measurement system analysis method on TD network insertion loss", Modern Manufacturing Engineering, vol. 4, no. 108, (2013).

[2] C. J. Shuai and T. J. Han, "Linear boundary element of angle arc method analysis on special transmission lines with offset inner conductor", Journal of Shaanxi University of Technology (Natural Science Edition), vol. 3, no. 18, (2010).

[3] J.S. Lin, Television Technology, vol. 12, no. 40, (2011).

[4] Y. M. Zhu and P. Guo and Y. T. Guo, Industrial Engineering And Management, vol. 3, no. 126, (2005).

[5] K. He, Course of Econometrics, Beijing: China Statistics Press, (2000).

[6] H. M. Fu and Q. Wu, Mechanical Strength, vol. 4, no. 600, (2010).

[7] C. J. Shuai and R. M. Li, "Study on empoasca vitis gothe egg incubation by non-thermal effect model", Journal of Chemical and Pharmaceutical Research, vol. 5, no. 47, (2014).

[8] R. M. Li and J. S. Zhang, "The professional technicians performance appraisal of coal mine", Coal Technology, vol. 7, no. 249, (2011).

[9] C. J. Shui and Y. Qian, "Finite-difference method analysis on the dissymmetrical double-ridge waveguide", Journal of Shaanxi University of Technology (Natural Science Edition), vol. 6, no. 11, (2013).

[10] R. M. Li, "The Company's Stock Price of Forecast with ARIMA", Shandong Youth, vol. 3, no. 137, (2013).

[11] C. Viroli, Journal of Multivariate Analysis, vol. 111, no. 296, (2012).

[12] Azar and A. Taher, "International Journal of Biomedical Engineering and Technology", vol. 2, no. 122 (2012).

[13] Nguyen and D. Cong, Azadivar. Journal of Medical Imaging and Health Informatics, vol. 3, no. 336, (2012).

[14] X. H .Han and Y. H. Ge, "Simple Linear Regression with Independent Variables Optimized", Engineering of Surveying and Mapping, vol. 3, no. 13, (2012).

[15] J. C. Mi, "Uncartainty Analysis of Unary Linear Regression”, Goal Quality Technology, 1, 21, (2014).

[16] L. C. Wu, Y. P. WU and Y. P. Xia, "Improvement of Significant Testing Method for a Linear Regression Model”, Geomatics \& Spatial Information Technology, vol. 2, no. 152, (2008).

[17] C. J. Shuai, "Analysis of Coupling Effectiveness on Concealed Signal Cable Slot with Different Shapes", Sensors \& Transducers, vol. 1, no. 219, (2014).

[18] C. J. Shuai, "Analysis on the 2D Electrostatic Field of Polygon Groove by MATLAB", Journal of Shaanxi University of Technology (Natural Science Edition), vol. 1, no. 59, (2012).

[19] C. J. Shuai, K. M. Teng and H. Jia, "On the Error Estimates of a New Operator Splitting Scheme for the Navier-Stokes Equations with Coriolis Force", Mathematical Problems in Engineering, Article ID 105735, 23 pages, vol. 12, (2012).

\section{Authors}

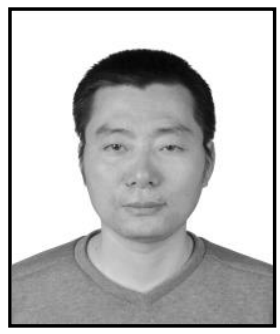

\section{Author's Name: Wei He}

Author's profile: Wei He (1972-), male, born in Shaanxi Hanzhong, lecturer, major: electronics and information engineering. 